\title{
ON THE DIPOLE-LIKE PROGRESSIVE WAVE \\ IN THE PHOTOSPHERE
}

\author{
I. K. CSADA
}

Konkoly Observatory, Budapest, Hungary

\begin{abstract}
Statistical analysis of the photospheric magnetic field averaged over 27 day time intervals shows periodical variations. The results of such an analysis are usually considered to be a pure mathematical representation of the observation material and additional analysis is necessary to infer the physical meaning of the results. Some ideas for this analysis are given by the mathematical method of the 'non-axisymmetric dynamo' theories which suggest the representation of the field by the series
\end{abstract}

$$
H_{x}=\sum_{n} \sum_{m=1}^{n} \mathscr{F}_{n m}(r, v) e^{i(\Omega+m \varphi)}
$$

i.e. the field variation is equivalent to the interference of progressive wave moving parallel to the equator with velocity

$$
|v|=\Omega / m \text {. }
$$

If $n=1$ the variation is equivalent to a progressive dipole field circulating over the photosphere by a time $2 \pi / \Omega$ and this period can be derived from the 27 -day averages. The superimposition of the circulation to the rotation will result in the apparent 'field rotation' being observable as recurrence time of the largest magnetic areas. Observational evidences are found for a progressive dipole circulating in a great circle with a period of $4 \frac{1}{6} \mathrm{yr}$.

The higher multipole field components (if $n \geq 2$ ) have no characteristic variations but their mean effect results in statistical spread in the 'multipole field rotation'. 